Fecha de recepción: diciembre 2017 Fecha de aceptación: octubre 2018 Versión final: julio 2019

\section{Exploraciones multimodales. Aportes para la enseñanza de la Comunicación Social}

\author{
Diego A. Moreiras * y Fabiana Castagno *2
}

\begin{abstract}
Resumen: Este escrito presenta una experiencia de trabajo en una cátedra del Profesorado en Comunicación Social de la Universidad Nacional de Córdoba, que es analizada a partir de los conceptos de exploración y multimodalidad. Se propone, a modo de marco teórico de la experiencia, un abordaje del campo de la multimodalidad que recupera ideas centrales para su consideración desde la comunicación social. En segundo lugar, se comparten los rasgos principales de esta experiencia en cuestión, así como una breve justificación teórico-didáctica para la misma y se presentan algunos resultados que involucran producciones efectuadas por estudiantes y sus propias voces. Finalmente, a partir de los desarrollos anteriores, se proponen orientaciones para acompañar la organización del trabajo de enseñar por parte de los (futuros) docentes en Comunicación Social, como un aporte a una didáctica de la comunicación en construcción.
\end{abstract}

Palabras clave: formación docente inicial - multimodalidad - exploración - didáctica de la comunicación.

[Resúmenes en inglés y portugués en la página 186]

${ }^{(*)}$ Diego A. Moreiras. Profesor en Primer y Segundo Ciclo de la EGB, Licenciado en Comunicación Social y Magister en Investigación Educativa. Docente del Profesorado en Comunicación Social, de la Facultad de Ciencias de la Comunicación e investigador de la Facultad de Ciencias Sociales, ambas de la Universidad Nacional de Córdoba. Sus temas de interés se relacionan con la comunicación / educación y enseñanza de la comunicación. Ha participado y codirigido equipos de investigación en estas temáticas. Doctorando en Semiótica, su trabajo de tesis en curso aborda las producciones audiovisuales en escuelas secundarias de Córdoba. Sus publicaciones abordan producciones fotográficas y audiovisuales en escuelas de Córdoba.

${ }^{\left({ }^{2}\right)}$ Fabiana Castagno. Profesora y Licenciada en Ciencias de la Educación. Docente del Profesorado y de la Licenciatura en Comunicación Social e investigadora de la Facultad de Ciencias de la Comunicación de la Universidad Nacional de Córdoba. Sus temas de interés se relacionan con la lectura y la escritura en procesos de formación en la educación superior. Ha dirigido proyectos y codirigido programas de investigación relacionados con la alfabetización académica y digital. Posee publicaciones sobre estas temáticas. Ha participado de proyectos interinstitucionales con otras universidades de Argentina y del 
exterior. Entre otros, la coordinación general del proyecto interdisciplinario de traducción y publicación del libro Escribir a través del currículum. Una guía de referencia (Bazerman y otros, 2016) y del Proyecto Writing Programs in Higher Education (Fulbright Specialist Program). Es co-fundadora de la Red Argentina de Instituciones Lectoras y Escritoras de Educación Superior.

\section{Presentación}

Durante las últimas décadas, profundas transformaciones que van de la mano de las tecnologías de la información y la comunicación impactan de manera profunda en las distintas esferas de la vida social, en las formas de apropiación, circulación y producción de conocimiento. Esto se evidencia en el campo educativo, y con particular énfasis en ámbitos formales, al interpelar y redefinir las formas tradicionales de enseñar y de aprender. Estos cambios involucran otros modos de representar y conocer el mundo. Numerosos estudios e investigaciones de diverso origen disciplinar -como la lingüística, la semiótica, la comunicación, la psicología, la antropología, entre otros- han aportado al análisis de estos fenómenos y han contribuido a poner en entredicho el lugar de la alfabetización en su sentido tradicional, sostenida en el dominio de la articulación libro-escritura y en una gramática escolar moderna, para ampliarlas y redefinirlas (como prácticas sociales situadas) en términos de alfabetizaciones múltiples que abren a una comprensión distinta de estos fenómenos (Martín Barbero, 1996, 2002; Cope y Kalantzis, 2009; Kress, 2000; Kress y Van Leeuwen, 2001, entre otros).

En ese marco, nos proponemos presentar algunos trazos de lo que podría considerarse una didáctica posible para la enseñanza de la comunicación social en la escuela secundaria, a partir de los desarrollos de una cátedra de grado, en el Profesorado en Comunicación Social de la Universidad Nacional de Córdoba ${ }^{1}$. En esta construcción hemos propuesto hasta aquí algunos conceptos que serían estructurantes de esta didáctica, como los de géneros (Bajtin, 2005; Bazerman, 2012, 2014, Bazerman y otros, 2016), lenguajes (Cope y Kalantzis, 2009, Bezemer y Kress, 2008) y traducciones (Lotman, 2009; Eco, 2008), presentados en una publicación anterior (Castagno, Pinque y Moreiras, 2015). En este escrito buscamos incorporar dos conceptos que entendemos la complejizan a la vez que nos permiten precisarla: nos referimos a los conceptos de multimodalidad y de exploración. Si aceptamos como hipótesis de trabajo que una pedagogía del diseño y de la multimodalidad puede realizar aportes en cualquier espacio curricular de la educación secundaria, como demuestran diversos trabajos publicados ${ }^{2}$, en el área de la comunicación social se torna doblemente pertinente. Se trata de promover estas experiencias de producción como un componente de la formación de base de los futuros docentes, hoy estudiantes del Profesorado y, según planteamos, como componente también de la multimodalidad y de una didáctica de la comunicación. En la primera parte de este escrito nos detendremos para justificar esta relación a modo de planteamiento teórico y de análisis de antecedentes. En relación con el segundo concepto, la idea de exploración es entendida en este escrito 
de la misma manera en que es presentada en la convocatoria: como una fase integrada y necesaria del proceso de producción, tanto en el trabajo profesional como en la formación de docentes. En el segundo apartado de este escrito sometemos a análisis la propuesta desarrollada como cátedra en el período entre 2014 y 2017, justamente a partir de la idea de exploración. Presentamos una descripción de la propuesta desarrollada, así como una breve justificación teórica para la misma, los objetivos que nos propusimos y algunos resultados obtenidos. Veremos allí que a partir de la exploración como categoría metodológico-didáctica proponemos un encuentro entre comunicación y multimodalidad. Finalmente, buscaremos compartir con el lector algunas ideas-fuerza para una didáctica de la comunicación (en construcción), que recupera el trabajo realizado hasta aquí bajo la forma de cuatro tesis para la elaboración de propuestas de enseñanza en este campo.

\section{Parte uno: Revisión teórica y de antecedentes}

\section{La multimodalidad en la enseñanza de la comunicación}

A partir de lo desarrollado hasta aquí, en este apartado nos interesa, en primer lugar, recuperar ideas fuertes de los escritos sobre multimodalidad para dar cuenta de sus aportes para la comprensión del campo de la comunicación en particular. En segundo lugar, realizaremos el movimiento inverso: desde el campo de la comunicación, recuperaremos autores y textos relevantes, de estrecha sintonía con la perspectiva de multimodalidad.

\section{1 ¿Qué elementos recuperamos de la multimodalidad?}

De forma sintética nos interesa proponer aquí un abordaje del campo de la multimodalidad que recupera tres ideas que consideramos centrales para su consideración desde la comunicación: a) la multimodalidad como característica de la construcción discursiva contemporánea; b) la multimodalidad como perspectiva ético-política con relación a los conocimientos y saberes; c) la multimodalidad como invitación a prácticas escolares de producción discursiva. A su vez, para cada una de ellas, proponemos sendas caracterizaciones que las recorren de modo transversal y resaltan ciertos rasgos que resultan fundamentales para este trabajo: la presencia de las tecnologías de la información y la comunicación (TIC) en nuestras sociedades contemporáneas; una posición ética y democrática frente al acceso y la construcción del conocimiento; la relevancia de lo que podríamos denominar pedagogías de la producción (discursiva), respectivamente. En relación con la multimodalidad, por lo tanto:

a. nos interesan dos ideas generales: construimos conocimientos a través de múltiples medios, como parte de procesos contextuales, situados (Luke, 2003) y cada día más los discursos permiten la construcción de sentido a través de la interacción de múltiples modos (Kress, Leite-García y Van Leeuwen, 1997). Esta dimensión de la multimodalidad en- 
cuentra, al menos en parte, su condición de posibilidad a partir de lo que Becerra (2003) enuncia como un entramado convergente de industrias info-comunicacionales: las de las telecomunicaciones, la microinformática y la audiovisual. Entre las tres, dan origen a las tecnologías y dispositivos que moldean nuestras vidas cotidianas hoy, así como los discursos y prácticas de ellas derivados. Por mencionar algunas: centralidad de la imagen, geolocalización, instantaneidad y ubicuidad. Y en esta dimensión, el lugar que ocupan (especial aunque no exclusivamente) los jóvenes y sus vínculos con estas tecnologías de la información y la comunicación.

b. resaltamos una perspectiva gnoseológica que permite considerar conceptualmente la construcción de conocimientos como relacionada pero no supeditada a la noción de literacidad. Y de hecho, permite "abrir" esta noción para dar cuenta de instancias de producción de sentido en recepción que no necesitan del código escrito de manera estricta. Esta dimensión apunta a la politicidad del saber y el reconocimiento de múltiples modos de acceder al conocimiento así como de compartirlo y ponerlo en circulación ${ }^{3}$.

c. asumimos una perspectiva pedagógica que, en los artículos que aportan conocimiento al campo, de manera explícita o no, afirma que los procesos asociados a la multimodalidad implican la creación, la recreación y la recontextualización, tal como es entendido este último concepto por Bezemer y Kress (2008). Así, en tercer lugar, esta dimensión resulta relevante ya que pone el foco sobre un modo particular de producción de conocimientos: aquel derivado de una práctica, de un "hacer", de una instancia de producción. A la vez, esta insistencia señala nuestra opción por ejercitar perspectivas pedagógicas que interpelen a los sujetos desde propuestas de enseñanza que se basan en experiencias escolares de producción de conocimiento.

\section{2 ¿Existen puntos de contacto entre los desarrollos de multimodalidad y el campo de la comunicación (y su enseñanza)?}

Además de lo señalado en el apartado anterior, nos interesa el campo de la multimodalidad toda vez que puede "ser leído" desde tradiciones y desarrollos propios del campo de la comunicación, especialmente desde Argentina. En el juego de establecer relaciones, proximidades y solapamientos entre ambos, lo que surge es la posibilidad de reorganizar un recorrido por el campo de la comunicación por parte de docentes en formación, así como ampliar los fundamentos para las decisiones que se toman en las prácticas de comunicación/ educación. A continuación, queremos contribuir a esos objetivos a partir de señalar algunas de estas proximidades, de manera sintética y esquemática:

- el sentido se construye tanto en producción como en recepción. En relación con esta idea podemos mencionar los trabajos pioneros de Jesús Martín Barbero (1987) y Stuart Hall (1993), así como los de los integrantes de la "primera" Escuela de Birmingham y en general, de los estudios de corte cualitativo que nos permitieron comprender, décadas atrás, que los espectadores y los lectores hacían cosas a partir de lo que los medios 
pretendían hacer con ellos. En nuestra región, estos desarrollos tuvieron su lugar en los escritos que permitieron cuestionar los modelos lineales de la comunicación, para prestar atención a la dimensión significante de los fenómenos sociales, a los procesos sociales de construcción de sentido. Junto al de Martín Barbero ya mencionado, los trabajos de Héctor Schmucler (1997) y María Cristina Mata (1985), por mencionar algunos, en las décadas del setenta y ochenta en nuestro país, son excelentes ejemplos.

- el sentido se produce y vehiculiza a partir de materias significantes y por lo tanto, estas, así como los lenguajes involucrados, tienen consecuencias en el "resultado final": diferentes modos y medios promueven la puesta en circulación de sentidos diversos. Eliseo Verón en La semiosis social (1987) abría la posibilidad de incorporar las materias significantes como un componente del análisis, a partir de una síntesis entre los modelos de producción del marxismo y las reflexiones sobre los modelos ternarios de producción de sentido, especialmente, el de Charles S. Peirce. En este esfuerzo de síntesis de Verón encontramos no sólo una salida a los desarrollos desde esquemas binarios de signo, y por tanto, la apertura a la reflexión sobre la producción del sentido (y no ya la reproducción de la relación significante-significado), sino una invitación a considerar las materias en las que el sentido es invertido como elemento constitutivo de estos estudios. Es por eso que no resulta difícil encontrar en los trabajos de este autor una consideración tanto de la materia lingüística, que no abandona ni desconsidera, como de otros modos y medios involucrados en la producción discursiva de los medios masivos de comunicación: fotografías, diseño de página, posición frente a la cámara, entre otras.

- la producción subjetiva y discursiva son procesos articulados. La idea de que existe una relación estrecha entre producción discursiva y producción subjetiva que encontramos en Kress (2000) es uno de los pilares del campo que Jorge Huergo denominó Comunicación/ Educación (cfr. Huergo 2001 y 2011). Esta forma de vincular ambos conceptos, tensionados a la vez que articulados por la barra, recupera la manera en que Héctor Schmucler (1997) concibió la relación entre comunicación/ cultura ${ }^{4}$. En este marco, así entendidas, ni comunicación remite tan sólo a los medios masivos ni educación queda confinada a la escolarización. Comunicación nombrará no sólo prácticas y procesos de producción discursiva, sino también prácticas y procesos que modelan sujetos (a partir de la actividad de los medios pero también de otros actores sociales); educación nombrará procesos de producción de sujetos pero también de producción de discursos, evidentemente (tanto desde escuelas como desde otras instituciones sociales). Se abandonan, tanto para la comunicación como para la educación, la inscripción de roles estáticos, unidireccionales en beneficio de una complejización en la interpretación de los procesos involucrados en ellas. Se abandona también la concepción ingenua que concentraba en las instancias de emisión (sean los medios como los docentes e instituciones) toda la capacidad de modelado y determinación, frente a interlocutores pasivos e, incluso, indefensos.

- La multimodalidad apunta a lo cotidiano, lo banal, lo ordinario. El campo de la comunicación fue pionero en los estudios que asumieron como objeto de investigación formas de la cultura consideradas hasta entonces como "menores". En nuestro país resultan espe- 
cialmente relevantes los estudios de Aníbal Ford, Jorge Rivera y Eduardo Romano (1987), articulados en torno a las nociones de medios de comunicación y cultura popular, título de uno de sus libros más emblemáticos. En esta opción reconocemos una preocupación nacida de una mirada atenta a los procesos desarrollados en la propia contemporaneidad, así como el convencimiento de que estos trabajos de indagación y reconstrucción de estos procesos eran relevantes e implicaban la invención de algo nuevo. Identificamos aquí una de las preocupaciones que Raymond Williams ponía en circulación en 1958: los significados más relevantes de una sociedad son aquellos que son comunes, compartidos por todos, "obra de todo un pueblo (...) fruto de toda la experiencia personal y social comprometida de un ser un humano" (Williams, 2008, p. 46). Lejos de cualquier sentido peyorativo, lo ordinario remite a lo común, a lo compartido por todos, a lo que forma parte de la vida cotidiana de las mayorías; esos son los procesos y prácticas que ameritan nuestra atención. En ese gesto político a la vez que académico reconocemos continuidades con los motores que impulsan los estudios de multimodalidad hoy.

\section{Parte dos: Descripción de la experiencia y resultados}

\section{Exploraciones multimodales en la enseñanza de la comunicación}

Presentaremos ahora la propuesta metodológica que sostuvo la experiencia de nuestra cátedra en el período correspondiente a los ciclos lectivos de 2014 a 2017. Participaron, según el año académico, un promedio de 40 estudiantes por cohorte. La tarea consistió globalmente en el análisis de experiencias y producciones - propias y ajenas- que involucraron una reflexión sistemática en torno a: diferentes lenguajes; diferentes géneros discursivos; las potencialidades de las TIC para presentar y representar de distintos modos saberes y conocimientos; la dimensión social/dialógica involucrada en estos procesos. En ese marco, la exploración estuvo concebida en tres planos: a) desde la creación por parte de la cátedra de situaciones y tareas que posibilitaran analizar experiencias y elaborar propuestas de naturaleza multimodal (como una fase central e ineludible del proceso de producción, dentro de una didáctica de la comunicación); b) como práctica estructurante de la enseñanza en el campo de la comunicación, toda vez que entendemos que los estudiantes del Profesorado tienen que vivenciar propuestas de formación que involucren exploración para apropiarse de ellas e implementarlas en su trabajo como docentes; y c) como posibilidad de traer al aula y a la vez enriquecer las trayectorias y los repertorios de los cursantes como profesionales ${ }^{5}$ y como sujetos de aprendizaje, semióticos y comunicacionales.

Nos interesa además recuperar algunas ideas de Eva Da Porta que acompañan teóricamente, junto a los desarrollos anteriores, las decisiones de la propuesta metodológica en nuestra cátedra. La autora afirma:

Experimentar [explorar] con los lenguajes mediáticos, con sus códigos, desarmar los mensajes, alterar sus modos tradicionales, explorar los géneros, desarticular los formatos típicos, utilizar la técnica como un modo de enriquecer la 
expresión, son quizás los aspectos más valiosos de estas experiencias (Da Porta, 2006, p. 172)

Este fragmento, si bien fue escrito en un contexto en que la comunicación social aún no era una orientación en sí misma en el sistema educativo argentino; en un momento en el que las tecnologías móviles de comunicación estaban recién comenzando su expansión y en el que la presencia en las escuelas de lo comunicativo tenía que ver sobre todo con los medios de comunicación; las orientaciones y abordajes que sugiere allí para el trabajo en escuelas siguen siendo completamente actuales y brindando buenas pistas para el mismo. Por último y de manera general, recuperamos desde el campo de la enseñanza, recomendaciones vinculadas tanto a la Escuela de Sidney como a enfoques constructivistas del aprendizaje con un fuerte acento en la exploración. Deseamos desde estos marcos generar actividades que los estudiantes transiten y que habiliten luego instancias de análisis y reflexión a partir de lo hecho. En particular, asumimos que el proceso de enseñanza y aprendizaje se entiende como un ciclo que parte de la deconstrucción grupal de determinados discursos/enunciados de interés, sigue con su producción conjunta y compartida, primero por fragmentos, luego de forma completa y culmina con su producción individual e independiente (Navarro y Revel Chion, 2013, p. 39 y 41).

A continuación describiremos brevemente las producciones solicitadas como así también algunos resultados.

\subsection{Algunas descripciones de la experiencia}

El primer momento, materializado en el Trabajo Práctico 1, tuvo una duración de un mes y fue de resolución grupal. Consistió en explorar distintas formas de representar un núcleo de saberes previos ligados a modelos, teorías o debates que conforman la comunicación como campo disciplinar. Esa experimentación se ancló en la recuperación de esos saberes a través de variadas formas de "decir", que habilitaron distintas posibilidades semióticas de pensar, comprender y comunicar ese saber disciplinar. Es decir, se buscó volver al campo de la comunicación, explorarlo o re-explorarlo y generar nuevos discursos, en diferentes modos y medios, que pudieran ser utilizados como materiales para la enseñanza en el nivel de destino (secundario). Estas tareas de análisis y producción exigieron una recontextualización/transformación de la comunicación desde objeto disciplinar hacia objeto de enseñanza.

Este trabajo constó de tres fases: 1) seleccionar, investigar y elaborar información para completar un cuadro comparativo multimodal sobre un modelo, teoría o debate relevante del campo de la comunicación; 2) traducir esa información para la producción de un texto que diera cuenta de ese modelo, teoría o debate a través de diferentes lenguajes y géneros solicitados: líneas de tiempo interactivas, mapas interactivos y multimedia, instructivos, glosarios y micro-programas de radio; 3 ) analizar y discutir colectivamente con el grupo clase tanto el proceso como las producciones resultantes. Las imágenes que presentamos a continuación corresponden al espacio de trabajo en el Aula Virtual de la Cátedra (Figura 1); al entorno de trabajo de las producciones de mapas interactivos (Figuras 2 y 3 ); y al 
repositorio de archivos sonoros en el cual fueron cargadas las producciones de microprogramas de radio (Figura 4). Debido a actualizaciones de las plataformas, se ha perdido acceso a las producciones de líneas de tiempo interactivas.

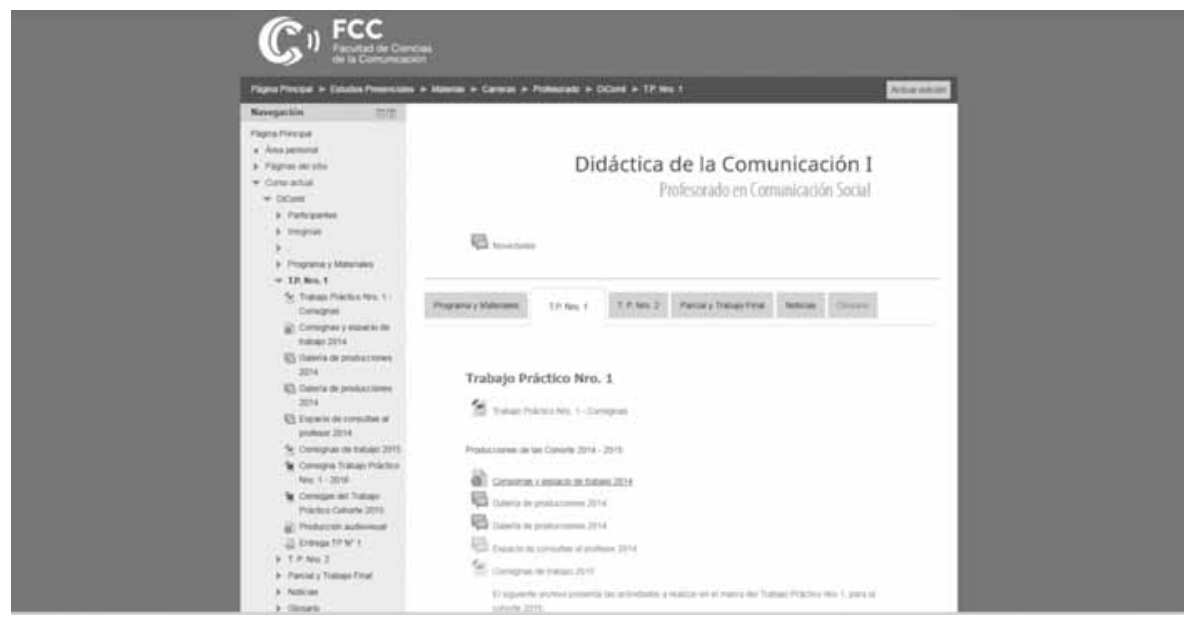

Figura 1. Pautas de Trabajo y organización del recorrido presentado en aula virtual.

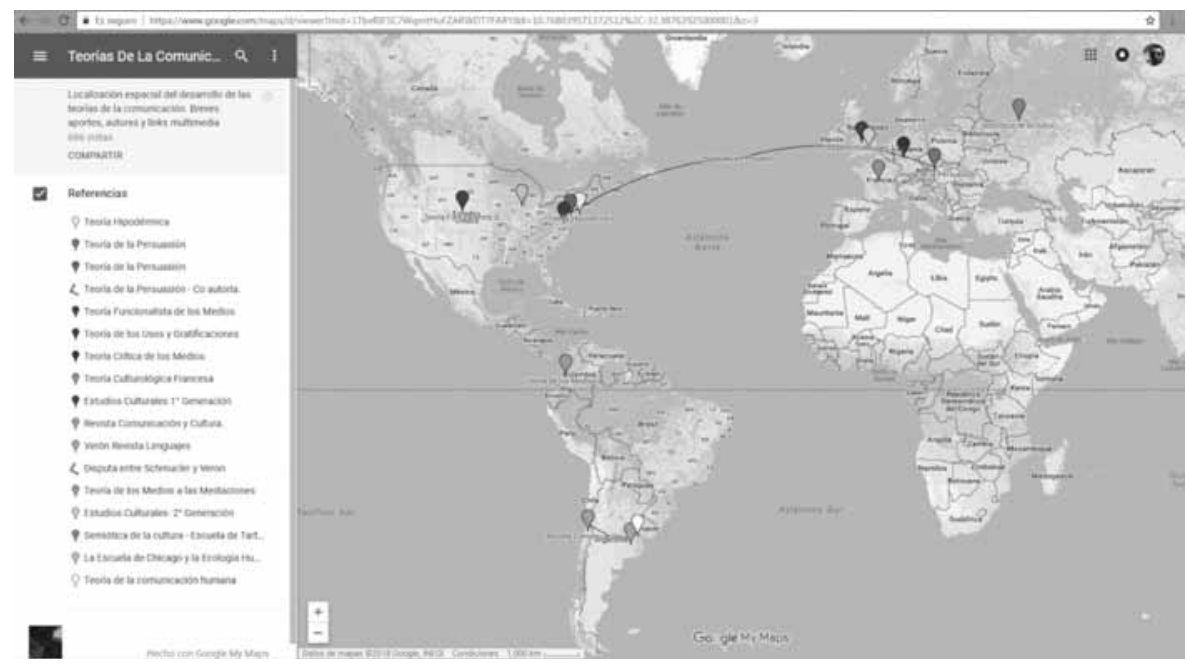

Figura 2. Mapa interactivo de presentación de las Teorías, Modelos y Debates relevantes del Campo de la Comunicación Social 


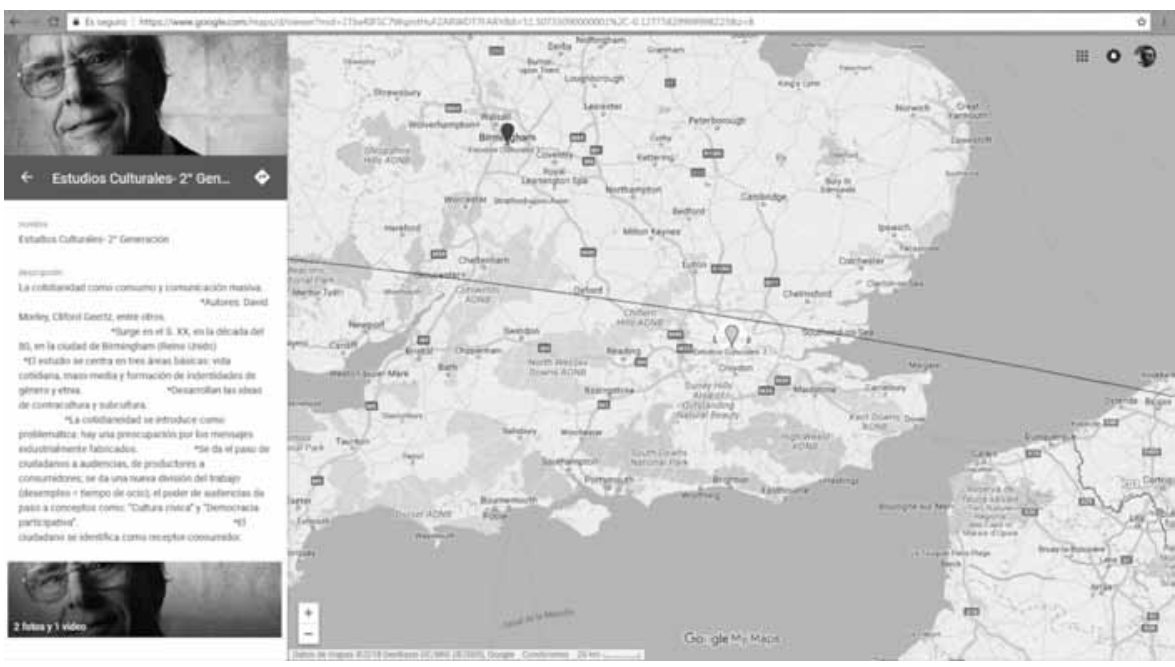

Figura 3. Un ejemplo de las propuestas recogidas en el Mapa interactivo de presentación de las Teorías, Modelos y Debates relevantes del Campo de la Comunicación Social.

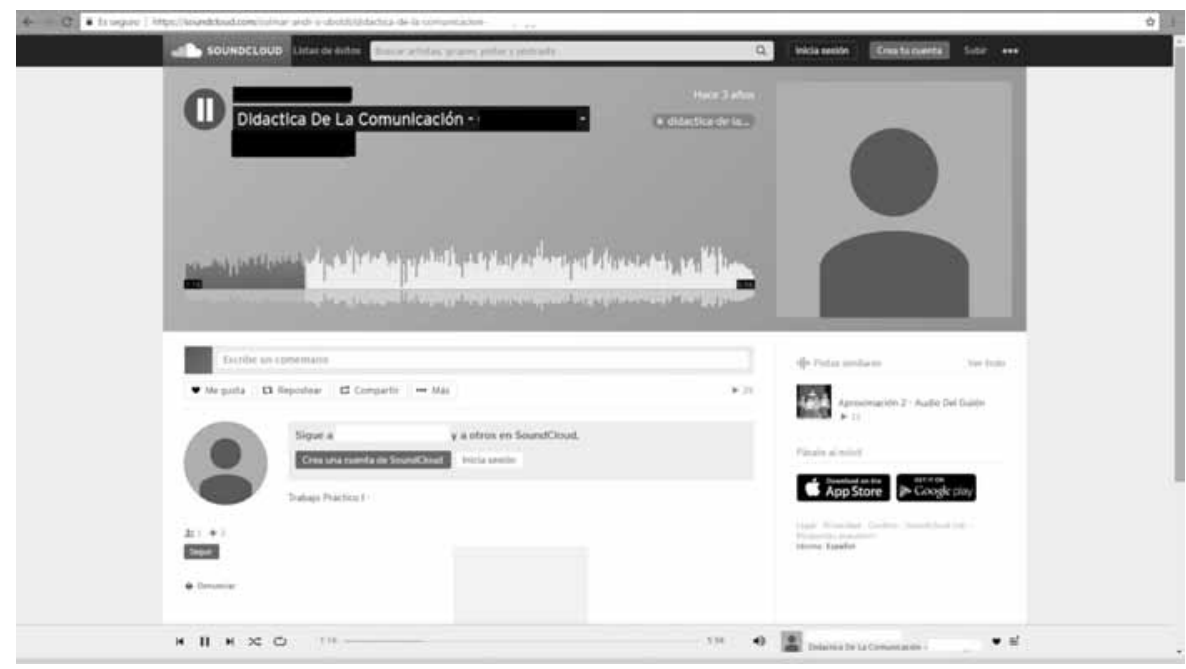

Figura 4. Producciones sonoras, bajo el géneros de micros radiales, de presentación de las Teorías, Modelos y Debates relevantes del Campo de la Comunicación Social 
El segundo momento, nucleado en torno al Trabajo Práctico 2, permitió un desplazamiento hacia el análisis de experiencias de otros: profesores -futuros pares- en el campo de la docencia. Las tareas involucraron tres etapas: 1) explorar y relevar, a partir de una guía proporcionada por la cátedra, propuestas y prácticas de enseñanza vinculadas al campo de la comunicación desarrolladas por profesores en escuelas secundarias de la ciudad de Córdoba o de localidades vecinas, 2) analizar claves genéricas de esas propuestas de enseñanza, para finalmente 3) efectuar alguna/s recomendación/es y sugerencias didáctica/s que pudieran ser útiles para ellos mismos al momento de la construcción de propuestas de enseñanza propias. Esta tarea demandó de los cursantes un doble movimiento: leer/ explorar una práctica concreta de un profesional en ejercicio en otro contexto, el de la escuela -potencial lugar de trabajo-, para escribir/ producir sugerencias sobre la base de criterios que informan la práctica profesional docente. Este trabajo tuvo una duración total similar al primero.

El tercer momento comprometió el desarrollo del Trabajo Final y supuso otro desplazamiento: hacia la posición de los cursantes como enseñantes. Implicó instancias evaluativas colectivas con el propósito de generar reflexiones y aportes para la construcción de propuestas de enseñanza y, mediatamente, de una didáctica de la comunicación. Las actividades tuvieron también tres etapas: 1) la producción de una pieza audiovisual que se plantea como un material didáctico con potenciales destinatarios: cursantes de la cátedra de la siguiente cohorte o estudiantes de educación secundaria, 2) la elaboración de un breve escrito que fundamente las decisiones de esa producción, 3) la presentación oral de la pieza audiovisual y el escrito de fundamentación en una Jornada de intercambio entre pares. Así, los estudiantes participaban de un género profesional en el cual se combinan -al menos- dos modos articulados: el audiovisual y el lingüístico, en el marco de una presentación oral. Estas producciones tematizan de modo explícito nuestro presente, sus conflictos y tensiones. Es habitual encontrar en ellas respuestas a preguntas sobre el nuevo rol docente que están construyendo (¿cómo incorporar las tecnologías en las aulas? ¿cómo construir autoridad en las aulas?) así como temáticas vinculadas a los jóvenes con los que trabajan o trabajarán: el uso y construcción de memes, el remixado como instancia de producción o el abuso policial y los casos de gatillo fácil en Córdoba. Entendemos que esta fase es de absoluta relevancia al promover un repertorio expandido para comprender y representar de manera distinta al menos dos planos de ese proceso: el contenido de la disciplina desde la perspectiva de la enseñanza y una aproximación a su desempeño como futuros docentes atravesado por la noción de hibridez ${ }^{6}$. De esta manera las tres producciones operan como un continuum que potencia la exploración de distintas formas de presentar y representar, al converger lo lingüístico, la imagen, el sonido y la gestualidad de los cuerpos en el marco de un sistema de actividades (Bazerman, 2012, Bazerman y otros, 2016 $)^{7}$. En otros términos, se diseñó un dispositivo de enseñanza que pudiera dar cuenta de una pedagogía de la producción multimodal situada. A modo de resumen de lo desarrollado hasta aquí, la Tabla 1 permite visualizar cómo se entrelazan exploración, multimodalidad y contextos a lo largo de la propuesta: 


\begin{tabular}{|c|c|c|c|}
\hline $\begin{array}{l}\text { Momento de la } \\
\text { experiencia }\end{array}$ & $\begin{array}{l}\text { Exploración y } \\
\text { Multimodalidad }\end{array}$ & $\begin{array}{l}\text { Primer contexto: } \\
\text { estudiantes de } \\
\text { Profesorado }\end{array}$ & $\begin{array}{l}\text { Segundo contexto: } \\
\text { estudiantes de } \\
\text { Secundaria }\end{array}$ \\
\hline $\begin{array}{l}\text { Primer momento: } \\
\text { producción discursiva } \\
\text { en torno al campo de } \\
\text { la comunicación. Un } \\
\text { mes. Por grupos. }\end{array}$ & $\begin{array}{l}\text { Re-exploración } \\
\text { del campo de } \\
\text { comunicación social; } \\
\text { producción discursiva } \\
\text { en múltiples modos y } \\
\text { medios; identificación } \\
\text { de temáticas } \\
\text { relevantes. }\end{array}$ & $\begin{array}{l}\text { Pasaje de la } \\
\text { comunicación como } \\
\text { disciplina a objeto } \\
\text { de enseñanza; } \\
\text { recuperación de } \\
\text { saberes profesionales } \\
\text { en nuevos contextos; } \\
\text { puesta en diálogo } \\
\text { con culturas juveniles. }\end{array}$ & \multirow{3}{*}{$\begin{array}{l}\text { Permiten pensar } \\
\text { instancias de } \\
\text { producción } \\
\text { discursiva, que } \\
\text { son también de } \\
\text { constitución subjetiva } \\
\text { y de construcción de } \\
\text { una voz y presencia } \\
\text { públicas (cfr. Da } \\
\text { Porta, 2006) } \\
\text { Se puede trabajar } \\
\text { sobre el cómo } \\
\text { "contar bien", } \\
\text { "mostrar bien", } \\
\text { "escribir bien" } \\
\text { poniendo el interés en } \\
\text { la comunicabilidad; } \\
\text { todas tareas propias } \\
\text { de la escuela. }\end{array}$} \\
\hline $\begin{array}{l}\text { Segundo momento: } \\
\text { relevamiento de } \\
\text { experiencias de } \\
\text { trabajo con TIC y } \\
\text { medios en escuelas } \\
\text { secundarias. Un mes. } \\
\text { Por grupos. }\end{array}$ & $\begin{array}{l}\text { Exploración de } \\
\text { claves genéricas: } \\
\text { construcción de } \\
\text { propuestas de } \\
\text { enseñanza, su } \\
\text { análisis, (auto) } \\
\text { sugerencias y (auto) } \\
\text { recomendaciones }\end{array}$ & $\begin{array}{l}\text { Recuperación de } \\
\text { saberes profesionales } \\
\text { (del campo de la } \\
\text { comunicación) } \\
\text { y creación / } \\
\text { consolidación de } \\
\text { aquellos propios } \\
\text { del campo de la } \\
\text { docencia. }\end{array}$ & \\
\hline $\begin{array}{l}\text { Tercer momento: } \\
\text { trabajo final } \\
\text { (audiovisual } \\
\text { más escrito y su } \\
\text { presentación oral). Un } \\
\text { mes. Por grupos y en } \\
\text { instancia colectiva. }\end{array}$ & $\begin{array}{l}\text { Exploración de } \\
\text { claves genéricas } \\
\text { de forma colectiva: } \\
\text { presentación, } \\
\text { exposición, síntesis } \\
\text { de ideas relevantes. }\end{array}$ & $\begin{array}{l}\text { Instancias evaluativas } \\
\text { colectivas que } \\
\text { buscan promover la } \\
\text { reflexión y generar } \\
\text { aportes para una } \\
\text { didáctica de la } \\
\text { comunicación. }\end{array}$ & \\
\hline
\end{tabular}

\subsection{Algunos resultados de la experiencia}

En esta sección presentaremos algunos resultados de la experiencia descripta en el apartado anterior sobre la base del análisis de producciones estudiantiles y valoraciones del proceso formativo por parte de los cursantes. Los datos fueron generados en el marco de la evaluación que el equipo de cátedra realiza de su propia práctica al final de cada uno de los semestres de clase.

- Acerca de las producciones estudiantiles

Cada año las producciones realizadas por los estudiantes amplían el horizonte de lo realizado previamente por las cohortes precedentes. En el cruce entre lenguajes (modos), 
géneros, tecnologías disponibles y escenarios de circulación, los discursos imaginados y desarrollados por los estudiantes dialogan con su contemporaneidad, con lo ordinario de la vida cotidiana de sus futuros estudiantes y también de sí mismos. Se animan a poner en circulación discursos que pueden ser resignificados de acuerdo a sus interlocutores, lo que supone asumir la complejidad de la enunciación multimedial hoy. Enuncian como objetivo explícito el trabajo con el conocimiento y los aprendizajes a construir por parte de los estudiantes secundarios con los que ya están trabajando o con los que se cruzarán en el futuro; o bien, se sitúan como enunciadores que dialogan horizontalmente con los compañeros de cohortes venideras al momento de elaborar materiales que imaginan propicios para nuestra cátedra del profesorado.

Hemos elegido para compartir en este artículo una de las producciones que se sitúan en este último grupo (Figura 5), debido a su disponibilidad en una plataforma de contenido audiovisual abierto garantizada por sus autores y porque consideramos que resulta un interesante ejemplo de los procesos de producción discursiva multimedial a los que hemos hecho referencia hasta aquí. Se denomina "Multiplicidad discursiva en el aula" y se puede visualizar en el siguiente enlace: https://www.youtube.com/watch?v=GINvv76lVjI

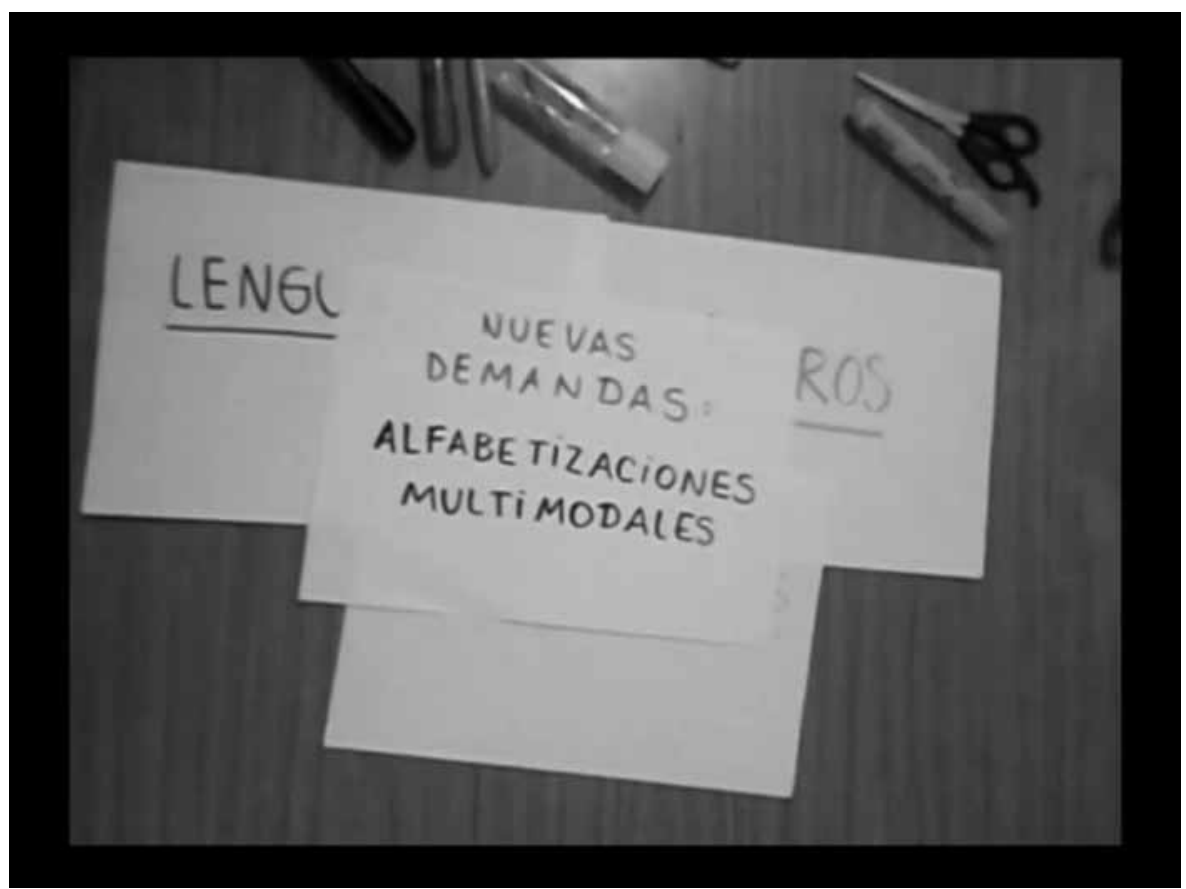

Figura 5. Captura de pantalla de la producción audiovisual "Multiplicidad discursiva en el aula”. Grupo: Alday, Losada, Oviedo y Payero. Año 2015. 
Nos interesa llamar la atención del lector sobre algunos de los aspectos que nos parecen relevantes en esta producción, sin pretender ser excluyentes:

- la construcción argumentativa del cortometraje, ya que plantea el caso de análisis (el Himno Nacional Argentino) desde el comienzo, para luego recuperarlo explícitamente como un ejemplo de los procesos operados por el mercado;

- su construcción enunciativa a partir de material recuperado de circulación social y también producido por ellos; un recurso "económico" como el de las placas que se colocan frente a cámara acompañadas por una voz en off, o el recurso al interrogante con el que finaliza, abriendo lugar a los debates en el aula;

- la construcción discursiva desde el montaje, que permite poner en diálogo espacios colectivos e individuales; personajes socialmente relevantes y actores de difícil acceso a los procesos de representación en los medios masivos, tanto como los idiomas en los que se comunican;

- la incorporación de conceptos e ideas trabajados en otros espacios (por ejemplo, los del aula universitaria), de manera que contemplan el nuevo esquema de circulación en el que son insertos, atendiendo a particularidades alrededor del lenguaje, los destinatarios, la intencionalidad y ciertos patrones estéticos;

- la centralidad y articulación en torno a lo auditivo y rítmico, por sobre otros lenguajes, lo que involucra, por transitividad, al cuerpo;

- la dimensión identitaria, tanto desde la referencia al Himno, como a ciertas prácticas, eventos culturales y la forma de vivirlos o tradiciones y lugares que nos son comunes.

Estas dimensiones así como otras que surgen de los propios estudiantes son las que constituyen los diálogos que propiciamos en el aula en torno a estas cuestiones.

Uno de los objetivos de estas propuestas, como ya dijimos antes, busca que los estudiantes logren sistematizar orientaciones y pautas surgidas del análisis de estas experiencias y que puedan convertirse en recomendaciones para su futuro trabajo docente. El hecho de que los objetos sobre los que se dialoga y reflexiona sean producciones realizadas por pares de la misma cohorte o de cohortes anteriores, resultado de recorridos muy personales de exploración a partir de pautas comunes, da lugar también a la puesta en valor de la idea misma de exploración como componente didáctico fundamental de estas experiencias. Nos interesa, a continuación, compartir algunas de las reflexiones surgidas en las evaluaciones realizadas cohorte a cohorte de lo trabajo durante el cursado. Estas reflexiones de los estudiantes complementan la presentación de resultados compartida hasta aquí.

\section{- Acerca de las valoraciones de los estudiantes}

Compartiremos algunas expresiones de los cursantes que entendemos dialogan con las ideas de multimodalidad y exploración para la enseñanza de la comunicación abordadas en la propuesta de la cátedra. Las mismas resultan del análisis de respuestas a cuestionarios semiestructurados autoadministrados en línea y anónimos a las cohortes comprendidas en cada uno de los períodos académicos mencionados (los años 2014 a 2017). Debido a la extensión de este trabajo, sólo retomaremos aquí datos obtenidos de preguntas abiertas a través de las cuales buscamos indagar la valoración de las producciones solicitadas. 
- La exploración de múltiples modos y medios para potenciar el aprender y enseñar Algunos cursantes reconocen la exploración multimodal como una posibilidad de experimentar y reflexionar acerca de las potencialidades de aprender y enseñar a partir de otras formas de representar, jerarquizar, comprender y pensar que habilitan diferentes lenguajes, géneros y los procesos de traducción comprometidos. Estas alusiones no sólo vinculan el par cognición/ representación sino también la relación cognición/ emoción, al incorporar, por ejemplo, la idea de conmoción, de disfrute, de inquietante. Expresiones estudiantiles que dan cuenta de lo señalado son:

Nos pareció super potente mostrar de otra manera -en este caso con imágenesesta realidad. Tratar de conmover, sensibilizar al otro seleccionando, jerarquizando, poniendo en juego la creatividad y los pocos o muchos recursos con los que contábamos, se volvió algo interesante. Aprender a mirar con otros ojos (...) (estudiante cohorte 2017).

Trabajar con la relación entre los conceptos lenguajes, géneros y traducciones sumado a una temática que me interpeló y poder generar un producto con la conjunción de ello me pareció muy valioso y lo disfruté (estudiante cohorte 2015).

Inquietante porque siempre me llevo a seguir haciéndome preguntas (estudiante cohorte 2017).

- La exploración como transformación/traducción

En algunos casos los cursantes destacan que la experiencia remite a ellos mismos como sujetos de exploración de sus propios saberes. Es decir, la exploración tiene también un componente reflexivo, un volver a mirar-se, escuchar-se, pensar-se (Larrosa, 1995). Por otro, reconocen como valiosa esa operación de revisar los saberes adquiridos en su formación previa para ser pensados en términos de enseñanza y, también, la inevitable transformación a que da lugar. Sobre el asunto dicen:

El trabajo en esta cátedra siempre tuvo una lógica distinta a las demás que cursábamos. Me hizo reencontrarme nuevamente con nociones que había dejado un tanto olvidadas (de la Licenciatura), entenderlas de otra forma (estudiante cohorte 2014).

(...) la producción del trabajo en sí requirió un nuevo re-acercamiento al campo de la comunicación y además nos planteó pensar y resignificar dicho campo y contenidos de ese campo para la enseñanza (estudiante cohorte 2016).

- La exploración multimodal como articulación entre producción discursiva y producción subjetiva dialógica

Para otros cursantes la experiencia significó la posibilidad de problematizar, de habilitar preguntas e indagar en nuevas claves genéricas propias del ámbito de la enseñanza. Se reconocen así las complejas relaciones entre producción discursiva y subjetiva a la vez que el contexto dialógico en que se suscitan: 
(...) permite pensar a la comunicación como objeto de enseñanza. Además, la instancia de Jornada es algo fuera de lo común, que posibilita que nos preparemos para una exposición con todo lo que eso requiere (algo que es frecuente en el trabajo docente) (estudiante cohorte 2015).

Fue muy interesante el modo cómo se abordó la materia, en momentos quizás hasta sorprendente (...), problematizar, poner en diálogo constante lo que vamos haciendo con lo realizado, construir y deconstruir para entender y aprender a ver operaciones que a veces no nos detenemos a pensar. Los múltiples lenguajes posibles para decir, comunicar, las potencialidades de cada uno, las relaciones entre didáctica y comunicación. La posibilidad de indagar, de ser creativo al momento de operar con traducciones y géneros (estudiante cohorte 2016).

Pensar en diferentes lenguajes habilita espacios creativos para que la enseñanza $y$ el aprendizaje sucedan (estudiante cohorte 2014)

En términos globales, entonces, las valoraciones de los estudiantes sobre la exploración multimodal permiten advertir la complejidad y multidimensionalidad a que dan lugar estos procesos al favorecer aprendizajes situados y colaborativos, sostenidos en la producción de sentido que abre a una comprensión diferente de sus experiencias y trayectorias como sujetos alfabetizados y alfabetizadores (Bazerman, 2014b).

En función de este recorrido propuesto, quisiéramos ahora, para finalizar, compartir algunas reflexiones finales bajo el formato de Tesis para una didáctica de la comunicación (en construcción).

\section{Parte tres: Propuesta para una didáctica de la comunicación}

A partir de los desarrollos anteriores, queremos proponer cuatro tesis para acompañar la organización del trabajo de enseñar por parte de los (futuros) docentes en comunicación social y que, a la vez, creemos que han organizado nuestra propuesta como cátedra hasta aquí. En ellas esperamos puedan reconocerse las huellas tanto del campo de la comunicación como del de la multimodalidad.

Tesis 1: Una didáctica de la comunicación debe trabajar explícitamente desde discursos multimodales. Una didáctica de la comunicación no puede trabajar sólo sobre el eje de la palabra, necesita del ingreso de otros lenguajes. Uno podría argumentar que es parte de las condiciones de la enseñanza hoy en cualquier espacio curricular la necesidad de atender a otros modos y medios y estamos de acuerdo. En el campo de la comunicación esto no se trata de una prescripción metodológica simplemente, sino a la vez de un contenido a ser enseñado. Por lo tanto, doble fundamento para esta consideración.

Tesis 2: Una didáctica de la comunicación invita a una reflexión en tiempo presente sobre la sociedad contemporánea. Esta reflexión puede articular todos los campos e incumbencias de la comunicación (desde el ejercicio del periodismo, hasta los desarrollos del marketing, la economía política de la comunicación o la investigación académica, por mencionar algunos), y sobre todo está en condiciones de anudar en esta reflexión la presencia de las TIC y sus posibilidades de creación y recreación discursiva, así como las 
transformaciones que ellas suponen en nuestras sociedades contemporáneas. Invita a una interrogación constante de los espacios y las prácticas más inmediatas que rodean a nuestros estudiantes. En esta reflexión creemos posible hacerle lugar a las tareas que posibiliten reconocer y analizar las instancias de enunciación y de recepción de discursos como articuladas, pero diferentes, así como la necesidad de desarrollar propuestas didácticas que las reconozcan, las diferencien y las analicen.

Tesis 3: Para una didáctica de la comunicación no hay saberes menores y no hay enunciadores menores. La historia del propio campo de la comunicación invita a prestar atención a discursos que tienen menor capacidad de circulación y de imponerse como legítimos, a enunciadores que tienen menos "prestigio", incluso a formas no tradicionales de construcción del conocimiento. A partir de este reconocimiento, la invitación es a pensar propuestas que permitan reflexionar sobre el poder, la hegemonía, el lugar de estos discursos y enunciadores en el ecosistema de medios, tanto como en la institución escolar.

Tesis 4: Una didáctica de la comunicación debe proponer transformaciones. En esta tesis recuperamos tanto la relación estrecha entre producción discursiva y producción subjetiva ya desarrollada, como la confianza en propuestas pedagógicas que articulen un "hacer" en la escuela, una exploración. La forma de entender a estas transformaciones guarda estrechas relaciones con la idea de traducción que mencionábamos antes, así como la de recontextualización, recuperada de Bezemer y Kress (2008). Además, sumamos aquí el esfuerzo necesario para generar en el aula propuestas de diferentes formatos curriculares y pedagógicos: pareciera que las clases no pueden ser ya (solamente) expositivas; es un desafío cotidiano promover otros formatos curriculares-pedagógicos, como talleres y seminarios, observatorios, laboratorios, ateneos, que pongan el foco en diferentes contextos, destinatarios, funciones e intencionalidades; que generen situaciones que permitan un "ponerse en contexto", un "hacer como si...", un "trabajar para otros".

\section{A modo de cierre}

Decíamos al comienzo que nos interesaba fundamentar la importancia de la multimodalidad y de la exploración para pensar los trabajos de docencia en comunicación en el plano de la formación inicial de docentes pero también en el de las aulas de educación secundaria en las que se desarrollarán nuestros estudiantes, futuros profesores. Sobre la base de la experiencia analizada, la exploración y la multimodalidad remiten a múltiples y complejos sentidos: la creación, lo que se "recuerda", lo que se "aprende". Además, involucran distintos planos tales como la cognición, la emoción, la interacción con otros, la situacionalidad, la materialidad, la representación y el sentido que los estudiantes reconocen que estas nociones han tenido para sus trayectorias de formación. Asimismo, les permiten ensayar propuestas para el trabajo en las escuelas secundarias de destino. Desde esta perspectiva, se advierte el potencial para diseñar proyectos de escrituras multimodales que posibiliten crear, recrear y recontextualizar saberes y prácticas cotidianos para desnaturalizarlos, desde una pedagogía de la producción situada.

Entre la pura reflexión teórica (alejada de las aulas) y la prescripción dura y automática (que desconoce particularidades y tiene escasa potencia creativa), deseamos que la siste- 
matización de prácticas que compartimos en este escrito pueda considerarse una contribución concreta al trabajo del docente en comunicación, que permita la planificación y la construcción de propuestas de enseñanza a partir de la articulación de campos y preocupaciones contemporáneas, como las que provienen de la multimodalidad y que entendemos contribuyen a delinear los contornos de una didáctica de la comunicación posible (y deseable), inquietud que mueve la presentación de esta experiencia.

\section{Notas}

1. Es importante aclarar que esta cátedra es parte de un Profesorado Ciclo, es decir, una carrera de formación docente destinada a egresados de la carrera de Comunicación Social de la misma casa de estudios, sea de la Tecnicatura o de la Licenciatura. Por lo tanto, los estudiantes con los que trabajamos son a la vez profesionales del campo de la comunicación social y algunos ya ejercen la docencia. Los trabajos de los que damos cuenta en este escrito han sido llevados adelante por los autores y por Germán Pinque y Yanina Maturo, como integrantes del Equipo de cátedra.

2. Por mencionar sólo algunos en nuestro idioma, consultar las publicaciones de Dominique Manghi, por ejemplo: Manghi Haquin (2011).

3. Un caso contundente son los desarrollos de este concepto en los estudios sobre educación de jóvenes y adultos, fundamentalmente en procesos de alfabetización inicial y educación primaria. Algunas ideas preliminares en este campo fueron recogidas en Moreiras (2015).

4. Schmucler entiende la articulación comunicación / cultura no a partir de una cópula que une pero al mismo tiempo afirma la distinción, sino a partir de la barra que mantiene ambos conceptos pero impide un tratamiento por separado. De esta manera, afirma la imposibilidad de pensar comunicación y cultura por separado.

5. Todos los ingresantes en este Profesorado son Técnicos Universitarios en Comunicación Social o Licenciados en Comunicación Social, ya que el profesorado ofrece una formación de complementación o Ciclo de Profesorado. Para el ingreso, es requisito indispensable haber obtenido el primero de los títulos mencionados. Esto implica que los estudiantes del Profesorado, en muchos casos, son profesionales del campo de la comunicación, que ejercen en diferentes esferas, organizaciones y entidades y cuyos saberes profesionales resultan por demás valiosos para el futuro ejercicio de la docencia.

6. Recuperamos aquí la idea de hibridez como mixtura que plantean Castelló, Banalez Faz y Vega López (2011).

7. La noción de cuerpo conformaría otra categoría distintiva para pensar una didáctica de este campo disciplinar, cuestión sobre la cual no ahondaremos por cuanto excede los límites de este trabajo.

\section{Referencias bibliográficas}

Bajtín, M. ([1982, 1979] 2005). El problema de los géneros discursivos. Estética de la creación verbal, Buenos Aires: Siglo XXI, pp. 248-293. 
Bazerman, C. (2012). Géneros textuales, tipificación y actividad. México: Benemérita Universidad de Puebla.

Bazerman, C. (2014a). La escritura en el mundo del conocimiento. Al encuentro de nuestra voz en la escuela, la universidad, la profesión y la sociedad. Verbum, 9(9), pp. 23-35.

Bazerman, C (2014b). Comprendiendo, un viaje que dura toda la vida: la evolución de la escritura. Infancia y Aprendizaje, 36 (4), pp. 421-441.

Bazerman, C.; Little, J., Bethel L., Chavkin, T., Fouqueette, D. y Garufis J. (2016). Navarro F. (Ed.) Escribir a través del curriculum. Una guía de referencia. Córdoba: Universidad Nacional de Córdoba.

Becerra, M. (2003). Sociedad de la información: proyecto, convergencia y divergencia. Buenos Aires: Grupo Editorial Norma. Enciclopedia Latinoamericana de Sociocultura y Comunicación.

Bezemer y Kress (2008). Writing in multimodal texts: a social semiotic account of design for learning. Written communication, 25 (2), pp. 166-195.

Castello, M., Bañales Faz, G. y Vega Lopez, N. (2011). Leer múltiples documentos para escribir textos académicos en la Universidad: o cómo aprender a leer y escribir en el lenguaje de las disciplinas. Revista Pro-Posicoes, Campiñas, V 22, n 1, pp. 97-114.

Castagno, F., Pinque, G. y Moreiras, D. (2015). "Sobre géneros, lenguajes y traducciones. ¿Hacia una didáctica de la comunicación?”. Revista El Cactus. Córdoba: Facultad de Ciencias de la Comunicación, Universidad Nacional de Córdoba, pp. 102-110.

Cope, B. y Kalantzis, M. (2009). “Multiliteracies”. New Literacies, New Learning, Pedagogies: An International Journal, 4:3, pp. 164-195.

Da Porta, E. (2006). Escuela y Medios: sentidos y sinsentidos. AAVV. Jóvenes. Identidad y Comunicación. En Proyecto Escuela, Universidad y Comunidad. Nuevas formas de relaciones. Córdoba: Min. de Educación de la Nación - ECI - Universidad Nacional de Córdoba, pp. 165-179.

Da Porta, E. (2011). Comunicación y Educación: algunas reflexiones para la búsqueda de nociones estratégicas. Da Porta, E. (comp) Comunicación y Educación. Debates actuales desde un campo estratégico. Córdoba: Gráfica del Sur, pp. 41-60.

Eco, U. ([2003] 2008). Decir casi lo mismo. Experiencias de traducción. Barcelona: Editorial Lumen.

Ford, A., Rivera, J. y Romano, E. (1987). Medios de comunicación y cultura popular. Buenos Aires: Ed. Legasa.

Hall, S. (1993). Encoding, decoding. En During, S. The cultural studies reader. London and New York: Routledge, pp. 90-103.

Huergo, J. (2001). Comunicación/Educación. Ámbitos, prácticas y perspectivas. La Plata: Ed. de Periodismo y Comunicación.

Huergo J. (2011). Sentidos estratégicos de comunicación/ educación en tiempos de restitución del Estado. Da Porta, E. (comp). Comunicación y Educación: debates actuales desde un campo estratégico. Córdoba: Gráfica del Sur, pp. 15-40.

Kress, G., Leite-García, R y Van Leeuwen, T. (1997). Discourse as structure and process. Discourse semiotics. Van Dijk, T. Discourse studies: a multidisciplinary introduction. Volume 1. London: SAGE. 
Kress, G. (2000). Multimodality: Challenges to thinking about language. TESOL Quarterly 34, Nro. 2. Teachers of English to Speakers of other languages Stable, pp. 337-340.

Kress, G. y Van Leeuwen, T. (2001). Multimodal discourse. The modes and media of contemporary communication. Oxford: Arnold.

Kress, G. (2005). El Alfabetismo en la era de los nuevos medios de comunicación. Granada: Consorcio Fernando de los Ríos.

Larrosa, J. (ed) (1995). Escuela, poder y subjetivación. Madrid: Ed. De La Piqueta.

Lotman, Y. M. (2009). Culture and explosion. Berlín: De Grouter Mouton.

Luke, C. (2003) Pedagogy, connectivity, multimodality and interdisciplinarity. Reading Research Quarterly, Vol. 38, Nro. 3. International Reading Association Stable, pp. 397-403.

Manghi Haquin, D. (2011). La perspectiva multimodal sobre la comunicación: desafíos y aportes para la enseñanza en el aula. Diálogos educativos, (22), pp. 3-14.

Mata M. C. (1985). Nociones para pensar la comunicación y la cultura masiva. En Curso de Especialización en Educación para la comunicación. Buenos Aires: Editorial La Crujía.

Martín Barbero, J. (1987). De los medios a las mediaciones. Barcelona: Gustavo Gili.

Martín Barbero, J. (1996). Heredando el futuro. Pensar la educación desde la comunicación. Revista Nómadas, No 5, Santa Fé de Bogotá (Colombia), pp. 10-22.

Martín Barbero, J. (2002). La educación desde la comunicación. Buenos Aires: Norma.

Moreiras, D. (2015). "Multimodalidad y Educación de Jóvenes y Adultos: Enfoque Teórico y Semiótica”, Ponce de La Fuente, H. y Dalmasso, M. (eds) Trayectos teóricos en Semiótica. Santiago de Chile: Facultad de Artes, Universidad de Chile y Centro de Estudios Avanzados, Universidad Nacional de Córdoba, pp. 139-147.

Navarro, F. (2013). Comunidades de práctica. En Diccionario de Nuevas formas de lectura y escritura. España: Santillana.

Navarro, F. (2014). Géneros discursivos e ingreso a las culturas disciplinares. Aportes para una didáctica de la lectura y la escritura. En Navarro, F. (coord.) Manual de escritura para carreras de humanidades. Buenos Aires: Facultad de Filosofía y Letras, Universidad Nacional de Buenos Aires, pp. 29-52.

Navarro, F. y Revel Chión, A. (2013). Escribir para aprender. Disciplinas y escritura en la escuela secundaria. Buenos Aires: Paidós.

Pinque, G. y Moreiras, D. (2015). “Acerca de lenguajes, géneros y traducciones. ¿Hacia una didáctica de la comunicación?”. Ponencia. VIII Seminario Regional (Cono Sur) ALAIC. 27 y 28 de agosto. Córdoba: Escuela de Ciencias de la Información, Universidad Nacional de Córdoba.

Schmucler, H. (1997). Memoria de la Comunicación. Buenos Aires: Ed. Biblos.

Verón, E. (1987). La semiosis social. Fragmentos de una teoría de la discursividad. Barcelona: Ed. Gedisa.

Wenger, E. (2001). Comunidades de práctica. Aprendizaje, significado e identidad. Buenos Aires: Paidós.

Williams, R. (2008). La cultura como algo ordinario. En García Ruiz, A. (ed.) Raymond Williams. Historia y cultura común. Madrid: La Catarata, pp. 37-62. 


\begin{abstract}
This text presents a teacher's experience in the Social Communication Teachers Program, in Universidad Nacional de Córdoba. We analyze this experience through the notions of exploration and multimodality. We propose, as a theoretical frame for this experience, an approach to the multimodality conceptual field, which recovers central ideas for the social communication field. In second turn, we share the main features of this experience and a brief theoretical and didactic approach to it. We also present productions made by the students, as well as their own voices on this process. Finally, considering the previous conceptual developments, we share some orientations that we find useful to organize the teaching work of (future) teachers in Social Communication, as a contribution to a didactics of social communication.
\end{abstract}

Keywords: initial teachers training - multimodality - exploration - didactics of social communication.

Resumo: Este artigo apresenta uma experiência de trabalho em uma Cátedra de Ensino de Comunicação Social na Universidade Nacional de Córdoba, que é analisada a partir dos conceitos de exploração e multimodalidade. Propõe-se, como referencial teórico da experiência, uma abordagem ao campo da multimodalidade que recupera ideias centrais para consideração da comunicação social. Em segundo lugar, são compartilhadas as principais características dessa experiência em questão, bem como uma breve justificativa teórico-didática, e são apresentados alguns resultados que envolvem produções feitas por alunos e por suas próprias vozes. Por fim, a partir dos desenvolvimentos anteriores, são propostas diretrizes para acompanhar a organização do trabalho docente pelos (futuros) professores em Comunicação Social, como contribuição para uma didática da comunicação na construção.

Palavras chave: Formação inicial de professores - multimodalidade - exploração - didática da comunicação.

[Las traducciones de los abstracts fueron supervisadas por el autor de cada artículo] 